\title{
Biology of HIV Transmission
}

National Cancer Institute

\section{Source}

National Cancer Institute. Biology of HIV Transmission. NCI Thesaurus. Code C19606.

Delineate the mechanisms of transmission and population spread of infection and their impact on disease progression. 